\section{P05 MULTIPLE RISK BEHAVIOUR IN ADOLESCENCE AND ADVERSE HEALTH AND SOCIAL OUTCOMES IN EARLY ADULTHOOD: FINDINGS FROM A PROSPECTIVE BIRTH COHORT STUDY}

${ }^{1} \mathrm{R}$ Campbell ${ }^{*},{ }^{1} \mathrm{M}$ Hickman, ${ }^{1} \mathrm{R}$ Kipping, ${ }^{2} \mathrm{M}$ Smith, ${ }^{3} \mathrm{~T}$ Pouliou, ${ }^{1} \mathrm{~J}$ Heron. ${ }^{1}$ School for Social and Community Medicine, University of Bristol, Bristol, UK; ${ }^{2}$ School for Clinical Medicine, University of Bristol, Bristol, UK; ${ }^{3}$ Administrative Data Research Wales, Swansea University, Swansea, UK

\subsection{6/jech-2017-SSMAbstracts. 107}

Background Adolescents engage in new and changed behaviours such as substance use or reduced physical activity. Evidence is accruing that these health risk behaviours co-occur at a population level and cluster within individuals. There has, however, been less focus on what the cumulative impact of engagement in multiple risk behaviours might be even in the short term. We sought to assess if engagement in multiple risk behaviours in mid-adolescence is associated with deleterious health and social outcomes in early adulthood.

Methods 5591 young people enrolled in the Avon Longitudinal Study of Parents and Children provided data on 13 risk behaviours from the domains of physical inactivity, substance use, self-harm, sexual health, vehicle-related risk ehavior, and antisocial ehavior when aged 15 to 16 . Logistic regression was used to examine any association between the total number of behaviours engaged in and rates of obesity, harmful alcohol use, problem gambling, anxiety, depression, trouble with the police and unemployment and lack of training at age 18 .

Results There was a strong association between multiple risk behaviour and all seven adverse outcomes. For each additional risk behaviour engaged in, the odds of having anxiety or being a problem gambler were $1.18[1.12,1.24]$ and 1.20 $[1.13,1.27]$ respectively, the odds for depression were 1.24 $[1.17,1.31]$, and the odds of getting into trouble with the police or of harmful drinking were 1.49 [1.42, 1.57] and 1.58 [1.48, 1.69] respectively. Adjustment for gender, parental socio-economic position, and maternal risk behaviours did little to alter the odds of these adverse outcomes. Confining analyses to adolescents not having experienced the adverse outcomes before age 15 , to exclude reverse causality, also produced little change in odds.

Conclusion Improvements in adolscent health are lagging behind those seen in child health. Investment in interventions and environments that effectively prevent multiple risk behaviour is likely to produce better adolescent health and wellbeing.

\section{P06 CORRELATES OF LEVEL AND LOSS OF GRIP STRENGTH IN LATER LIFE: FINDINGS FROM THE ENGLISH LONGITUDINAL STUDY OF AGEING AND THE HERTFORDSHIRE COHORT STUDY}

${ }^{1}$ LD Westbury*, ${ }^{1} \mathrm{HE}$ Syddall, ${ }^{1,2} \mathrm{EM}$ Dennison, ${ }^{1,3,4} \mathrm{C}$ Cooper, ${ }^{1,5} \mathrm{CR}$ Gale. ${ }^{1} \mathrm{MRC}$ Lifecourse Epidemiology Unit, University of Southampton, Southampton, UK; ${ }^{2}$ Victoria University of Wellington, Wellington, New Zealand; ${ }^{3}$ NIHR Southampton Biomedical Research Centre, University of Southampton and University Hospital Southampton NHS Foundation Trust, Southampton, UK; ${ }^{4}$ NIHR Musculoskeletal Biomedical Research Unit, University of Oxford, Oxford, UK; ${ }^{5}$ Centre for Cognitive Ageing and Cognitive Epidemiology, Department of Psychology, University of Edinburgh, Edinburgh, UK

10.1136/jech-2017-SSMAbstracts. 108
Background Low grip strength in later life is a risk factor for subsequent disability and mortality and a key component of sarcopenia. Established determinants of low grip strength level include: older age; shorter stature; poor nutrition; low physical activity; socio-economic disadvantage and multimorbidity. However, little is known about risk factors for accelerated loss of grip strength in later life.

Methods We examined socio-demographic, lifestyle and clinical predictors of baseline level and 8 year loss of grip strength in 3703 men and women (aged 52-82 years at baseline) in the English Longitudinal Study of Ageing (ELSA). Data on 441 men and women (aged 59-71 years at baseline) who participated in a 10 year follow-up of the Hertfordshire Cohort Study (HCS) were used for replication. Change in grip strength from baseline to the end of follow-up was characterised using linear mixed-effects models in ELSA as grip strength was measured at three time-points. A residual change method was used in HCS to obtain a measure of change in grip strength between two time-points which was independent of baseline level. Linear regression models adjusted for age and gender, as well as mutually-adjusted models, were used to examine the relationship between baseline predictors and level and change in grip strength in both cohorts.

Results Men in ELSA and HCS had higher average levels of grip strength at baseline, and accelerated rates of loss, compared with women. In ELSA, older age, shorter stature, and increased morbidity were associated with lower level, and accelerated rate of loss, of grip strength in both sexes. For example, accelerated loss of 0.04 (95\%CI: 0.00, 0.08) standard deviation (SD) scores per additional morbidity were estimated from mutually-adjusted models; corresponding SD scores per SD decrease in height were 0.06 (95\%CI: 0.03 , 0.09). In mutually-adjusted analyses, socioeconomic disadvantage, low level of physical activity and poorer self-reported health were also associated with low grip strength level, but not with rate of loss. Analysis in HCS yielded similar results.

Conclusion Our results identify multimorbidity as a modifiable determinant of both level and loss of muscle strength in later life. The association between shorter stature and accelerated loss of grip strength suggests that developmental influences may also impact on rate of loss, as well as on level, of muscle strength in older age. This research will inform the development of lifecourse interventions to promote maintenance, and reduce loss, of muscle strength in later life.

\section{P07 SENSORY IMPAIRMENTS AND COGNITIVE AGEING: FINDINGS FROM 11 EUROPEAN COUNTRIES}

A Maharani*, N Pendleton, G Tampubolon, J Nazroo, P Dawes. SENSE-Cog project, University of Manchester, Manchester, UK

\subsection{6/jech-2017-SSMAbstracts.109}

Background Sensory impairment (hearing and/or visual) and cognitive decline commonly occur in the elderly. Whether they are in fact associated, and whether sensory impairment might contribute to intellectual decline, has been a subject of a number of investigations during the past three decades. The purpose of this study was to assess in an older European population: (1) any independent association between single and dual sensory impairment (hearing and/or vision) and cognitive decline; (2) cognitive trajectories according to the pattern of sensory impairment. 